\title{
Design and Research job altitude four-wheeled vehicle control system Yinbo Du ${ }^{1, a}$, Li Tan ${ }^{1, b}$, Xian Meng ${ }^{1, b}$, Hong Xiang ${ }^{1, b}$, Dahong Wang ${ }^{2, c}$ \\ ${ }^{1}$ State Grid Chongqing Electric Power CO. Electric Power Research Institute, Chongqing 404100; ${ }^{2}$ Shenzhen Institute of Advanced Smart Technology, Shenzhen 518057 \\ 2,c903110232@qq.com
}

Keywords: four-wheeled vehicle, ARM, FPGA, PID.

\begin{abstract}
This paper introduces a flexibility and information on ARM + FPGA control platform, modular multi-sensor fusion, servo system as a dynamic system of aerial work four-wheeled vehicle solutions, the design of the control system to solve the possible interaction of control issues.
\end{abstract}

\section{Introduction}

System Components. Vehicle drive system configured as follows: front wheel hub directly drive a permanent magnet synchronous motors, compact, easy arrangement of the steering system. Rear wheel fixed a reducer on permanent magnet synchronous motors, can play a high-speed motor efficiency, the comprehensive advantages of high power density and high torque density of the system. At the same time, in order to effectively suppress the negative effects of the unsprung mass of the transmission and suspension arm integrated design. The basic vehicle design to meet the high-altitude electrified operation the wagon as a special project vehicles, the characteristics of the required high security, high adaptability, straightforward and efficient, high adaptability.

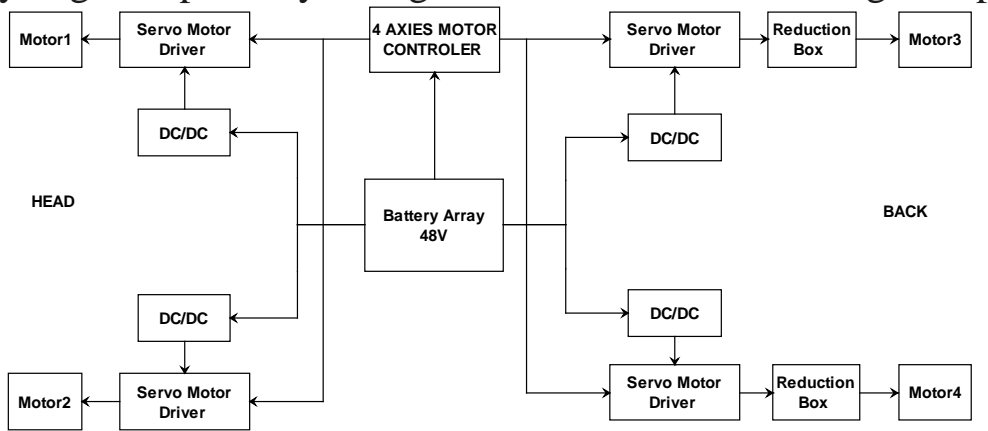

FIG1: System Components

Control Model. Driver model is actually a speed controller, as shown in the model using a PID controller is shown in Figure 2, input the desired speed and the actual vehicle speed $u_{d}, u_{a}$ (from the vehicle dynamics model output feedback) the difference $\Delta u$ into the accelerator pedal or brake pedal command $\beta_{\text {acc }}$ command $\beta_{\text {brk }}$. The main variables driver model for driver's weight, and the description is not With the driving style of $\mathrm{P}, \mathrm{I}, \mathrm{D}$ parameters.



FIG2: PID Driver model

The target vehicle speed and the actual vehicle speed deviation proportional, integral and differential control of the vehicle by a linear combination of demand-driven torque and braking torque to simulate the driver stepped on the accelerator pedal and the brake pedal of the process that controls the torque expression like (1) shown in the formula.

$$
T=k_{\mathrm{P}} e(t)+\frac{1}{T_{\mathrm{I}}} \int_{0}^{t} e(t) \mathrm{d} t+T_{\mathrm{D}} \frac{\mathrm{d} e(t)}{\mathrm{d} t}
$$

Wherein: $k_{p}$ is a proportionality coefficient; the $T_{I}$ is the integral time constant; $T_{D}$ is the derivative constant; e ( $\mathrm{t}$ ) as the deviation between the target vehicle speed and actual vehicle speed. 
Torque distribution control strategy based on the specific model, the model will control the torque distribution system demand torque distribution to the front wheels and the rear wheel hub motor wheel motor includes a drive torque distribution and braking torque distribution. The more common method is the basic principle adopted ,FOC control. Key vector control is still space current vector magnitude and phase (frequency and phase) of its control over the conversion relationship Fig 4.
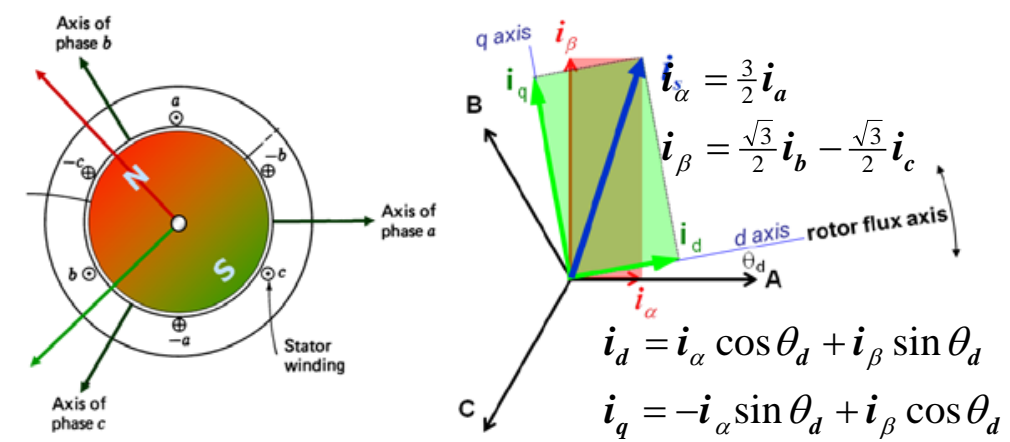

Fig 3: Field-oriented rotor axis system

Fig 4:The conversion relationship

\section{Control System Design}

Hardware Design. This system powered by 48V lithium battery drive four SYNTRON servo systems, 60LCB040C-J00000, which uses FOC algorithm control system has good and stable high speed performance, superior performance, speed and constant torque control performance is a good choice for four control systems. Therefore, the system use for direction of realize, speed mode and torque mode .

The motion controller uses ARM+FPGA design, using of ST high-performance 32-bit Cortex-M3 core STM32F103VC microprocessor, clocked at up to $72 \mathrm{MHz}$, and includes a rich peripheral interfaces; and using the latest fourth generation Cyclone TM FPGA EP4CE6E22C8N, by FSMC parallel bus combine ARM and FPGA. ARM as the main processor, FPGA as a coprocessor to complete the parallel event processing, the ARM rich interfaces and real-time FPGA combine complementary advantage control system. Design of a four-axis motion control, four servo motor drives are controlled four-wheel vehicle design diagram below FIG 5.

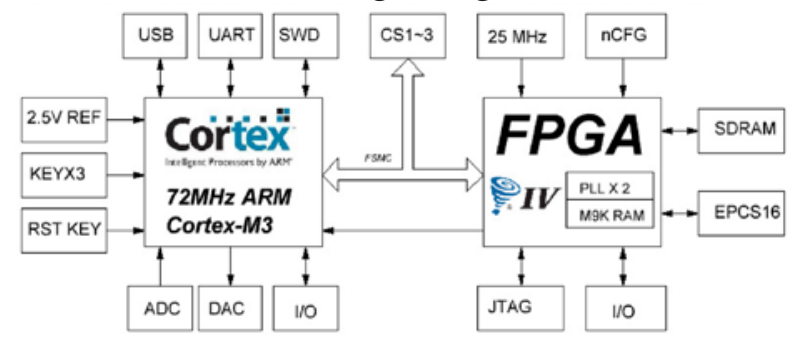

FIG 5: Controlled four-wheel vehicle design diagram

System Communication main two options: using ARM with CAN bus controller to communicate between each module through normal expansion IO port, using transformer isolation between the module using FPGA to build a path in SRAM design.

FPGA's IO was isolated inputs and outputs by optocoupler for control the servo system, speed pulse, enable control, alarm and fault clearing. SDRAM is for caching data. FPGA provides a $72 \mathrm{MHz}$ external clock input of ARM. ADC is mainly used to receive relevant analog sensor. DAC to implement torque control servo system. KEY and extended UART interface for serial communications, mainly for preliminary model debugging. ARM expansion I / O to limit switch inputs.

Software Design. By speed, torque and given feedback to obtain the corresponding error, and eliminate errors by the error, and ultimately achieve steady speed, torque output, PID control program flow FIG 6.

$$
u_{k}=K p * e_{k}+K i \sum_{j=0}^{k} e_{j}+K d\left(e_{k}-e_{k-1}\right)
$$


$\mathrm{K}$ is a sample sequence number, $\mathrm{U}_{\mathrm{k}}$ is the $\mathrm{K}$ times sampling time computer output, $\mathrm{e}_{\mathrm{k}}$ first $\mathrm{K}$ times the sampling time offset value input, $\mathrm{e}_{\mathrm{k}-1}$ the $\mathrm{k}-1$ times error sampling time of the input value $\mathrm{K}_{\mathrm{i}}$ is the integral constant, a $\mathrm{K}_{\mathrm{d}}$ of constant differential, $\mathrm{K}_{\mathrm{p}}$ is a proportional constant.
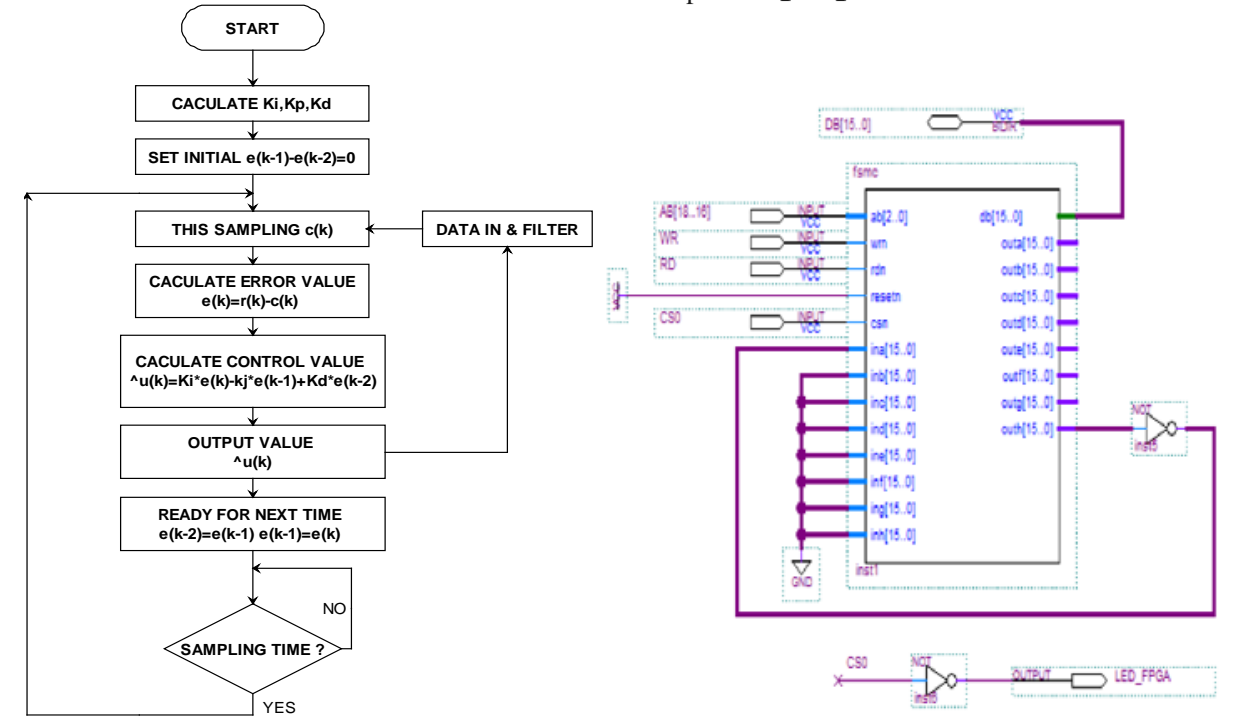

FIG 6: PID control program flow FIG 7: Top module connection diagram

Peripheral control: which simultaneously involves a lot of IO operation. Relative ARM and visits FPGA through FSMC using pointer operations, direct address access.

ARM write and read function:

\#define fpga_write (offset, data) * ((volatile unsigned short int *) (CS0_BASE + (offset $<<17))$ )

$=$ data.

\#define fpga_read (offset) $\quad *$ ((volatile unsigned short int *) (CS0_BASE + (offset $<<17)))$

Wherein:

offset $\quad$ spatial offset range of 0 to 7 (8 spaces).

data 16-bit integer in the range of $0 \sim 65535$.

CS0_BASE connection for ARM FPGA cs0 base address.

CAN node Software design are broadcast communication via CAN bus network between the hybrid drive system is discussed, the various subsystems and control units, as well as the power unit control unit.

FPGA software: Shown FMSC code generation top module connection diagram,

Among them:

DB [15..0] AB [18..16] WR RD CS0 is connected to the ARM.

LED_FPGA used to communicate instructions.

ina [15..0] inh [15..0] to read data input, contains a total of eight volumes, each 16.

outa [15..0] outh [15..0] to write register into space, a eight volumes, each 16 .

\section{Summary}

In this paper, the key issues in the four-wheeled vehicle control system design carried out in-depth theoretical study and gives wagon model, control model, the motor drive control method. A detailed description, and based on an ARM + FPGA modular control platform for multi-sensor fusion of four relatively practical universal car control scheme, the system acts as a reference demonstration.

\section{References}

[1]. Kiyomoto Kawakami, Kikuo Emoto, Takahisa Kamikura, et al. Evaluation of an ultra high performance EV'Eliica'. The $21^{\text {st }}$ International Battery,Hybrid and Fuel Cell Electric Vehicle Symposium\&Exposition.Monaco,2005. p.2-3. 
[2]. Xu Dan, Wang Guodong, Cao Binggang, Feng Xiaohu. Study on optimizing Torque Distribution Strategy for Independent 4WD Electric Vehicle. Journal of xi'an JiaoTong university Vo46 (2012) No.3, p. 43-46.

[3]. Li Weimin. RESEARCH OF HYBRID ELECTRIC VEHICLE CONTROL SYSTEM AND ENERGY MANAGEMENT STRATEGY. (Ph.D, Shanghai Jiao Tong University). p. 20-21. 